\title{
Cylinder-Cylinder Attraction in Uniform Flow
}

\section{Kern E. Kenyon}

4632 North Lane, Del Mar, USA

Correspondence to: Kern E. Kenyon, kernken@aol.com

Keywords: Flow Past Two Cylinders, Cylinder-Cylinder Attraction

Received: February 10, 2018 Accepted: March 9, 2018 Published: March 12, 2018

Copyright $\odot 2018$ by author and Scientific Research Publishing Inc.

This work is licensed under the Creative Commons Attribution International License (CC BY 4.0).

http://creativecommons.org/licenses/by/4.0/

\section{(c) (i) Open Access}

\section{ABSTRACT}

Two cylinders in a uniform flow can attract each other when their separation distance is within the zones of the perturbed velocities of the cylinders. Enhanced perturbed velocity between cylinders causes lowered pressure by Bernoulli's law. If the cylinders touch or the gap is very small, the flow will work to separate the cylinders. One result could be an oscillation. Two recent theoretical discussions of uniform and shear flows past a single cylinder helped spark the present prediction. No relevant experimental studies have been carried out so far.

\section{INTRODUCTION}

Undoubtedly the essence of this report could have been written up for dissemination many years ago. What has initiated doing so now is based on combining results of two recent and more or less elementary theoretical works on flows passing by cylinders, leaving plenty of room for future experimental comparisons that have so far not been obtained. One is a theory of the uniform flow past a cylinder that is not based on the traditional irrotational assumption (i.e. no potential flow dynamics are used) [1], and the other is a calculation of the lift force on a cylinder embedded in a shear current [2]. Both are believed to be new contributions.

When a uniform steady flow passes not too quickly by a fixed cylinder, a sheared current is created near the cylinder, according to observations and to two available theories. At the top and bottom surfaces of the cylinder, the flow speed is the greatest, and with increasing distance from the cylinder, the speed decays back to the uniform flow value far away. Attached to the cylinder's top and bottom sides are boundary layers of perturbed flow. If a small "test" cylinder is placed and held parallel within the shear zone of the larger cylinder, it is forecast to be attracted to the larger cylinder due to a pressure force related, through Bernoulli's law, to the difference is flow speed, caused by the larger cylinder, across the diameter of the test cylinder. That is the subject of this report.

\section{ATTRACTION CONCEPT}

Using a "test" cylinder for illustration is just a way to get the discussion started; it is not necessary. 
Two cylinders of equal size will be attracted to each other in a uniform flow provided they are within a certain range of each other. Too large a separation will not work nor will too small of a gap be affective.

Whether or not the potential flow theory has ever been applied to the problem of two cylinders in a uniform flow is not known to me, but I doubt that it has, for one reason, because of the mathematical complexities that would be involved. Also no apparent practical needs are waiting around that would justify expending such an effort. However, an alternative independent theory is available that might more easily be adapted to this particular situation.

In the new theory of a uniform flow past a single cylinder, along a line passing through the cylinder's center and perpendicular to the flow far away, there are two equations in the two unknowns: pressure and velocity. One equation is Bernoulli's law along a streamline. The second equation is the force balance on fluid particles traveling on curved streamlines; centrifugal force equals a pressure gradient. Each equation by itself is nonlinear. But when one equation in one unknown is formed, it is linear, both for pressure and for velocity! There is a non-constant coefficient, the radius of curvature of the streamlines as a function of distance away from the cylinder, that has to be supplied from theory or measurements, and then the integration of the one governing equation in one unknown can be completed. Linearity is such a big advantage that the inconvenience of the non-constant coefficient is surely only a small price to pay.

Linearity of equations implies that superposition of solutions will be possible, which opens a way to start understanding what happens when two cylinders are placed in a uniform flow. Since the increased perturbed flow over the top surface of one cylinder is presumed to add to the increased perturbed flow on the bottom surface of the second cylinder, which is located a certain distance above the first cylinder, the result is in a compounding of the total perturbed velocity, in speed and direction. Evidently the linear theory behind this prediction is bound to fail when the two cylinders are touching or very close together. Exactly where the failure can be considered to have occurred is not known a priori.

In conclusion, adopting the features of the linear theory helps along the prediction that between two cylinders, each being inside the perturbed velocity zone of the other, the velocity is greater than it would be if there were just a single cylinder, and it is also greater than that on the opposite sides of the cylinders. By Bernoulli's law then (where the speed is greatest the pressure is least) there is a net pressure force tending to decrease the distance between cylinders (until they get too close together, whatever that means).

\section{DISCUSSION}

To calculate the attractive force between two separated cylinders in a uniform flow, in algebraic language for example, the best starting point is the linear theory. This has not been done here but no major obstacles are foreseen to intervene in carrying it through.

Suppose the initial position of two cylinders is side by side, touching and facing broadside toward the mean flow direction. Then the flow will tend to pull the cylinders apart. But once a sufficient gap is formed, the flow will try to bring the cylinders back together again. A type of oscillation becomes possible under the right conditions. Has a partial or alternative explanation of some types of wind chimes just emerged? Additional work can be done along this avenue to establish the characteristics needed for an oscillation to occur from which more practical applications may follow besides the amusement of the wind chime, or perhaps also the annoyance of the rope snapping against the flag pole when there is no flag.

\section{REFERENCES}

1. Kenyon, K.E. (2013) Flow Past a Cylinder. Journal of Scientific Theory and Methods, 2103, 211-222.

2. Kenyon, K.E. (2017) Lift Force on a Cylinder in a Shear Current. European International Journal of Science and Technology, 6. 